\title{
Regional Signal-Delay Analysis Applied to High-Frequency Carbon Nanotube FETs
}

\author{
David L. Pulfrey, Fellow, IEEE, Leonardo C. Castro, David L. John, Member, IEEE, and \\ Mani Vaidyanathan, Member, IEEE
}

\begin{abstract}
A regional signal-delay analysis is presented for fieldeffect transistors intended for operation at very high frequencies. For the example used here of a doped-contact carbon nanotube field-effect transistor, the analysis reveals that tunneling into the channel region of the device, and modulation of the space-charge regions in the source and drain adjacent to the channel, are the principal contributors to the overall delay. A recently proposed lower limit to the signal delay time in the channel is critically examined via the introduction of a local signal velocity.
\end{abstract}

Index Terms-Carbon nanotube, field-effect transistors, high frequency, signal delay.

\section{INTRODUCTION}

$\mathbf{T}$ HE SMALL size, unusual topography, and technological immaturity of carbon nanotube field-effect transistors (CNFETs) have contributed, no doubt, to the fact that the present record for their measured unity-current-gain frequency $f_{T}$ is a modest $30 \mathrm{GHz}$ [1]. For the moment, then, we need to rely on simulations to get a better idea of the high-frequency capability of CNFETs. However, for the simulation results to be useful, the entire transistor must be considered, not just the gated region (channel) of the device. To neglect other regions where charge changes occur is, in an equivalent-circuit sense, to ignore the extrinsic capacitances [2]. Inclusion of such capacitances in simulations of switching speed [3], for example, indicates that considerable design optimization will be necessary if CNFETs are to realize the intrinsic advantage over Si MOSFETs that they are predicted to possess [4].

In this work, we focus on simulations of small-signal, highfrequency performance, and we apply to FETs the regionaldelay approach that has long been used as a tool to guide the development of high-frequency bipolar transistors [5], [6]. The method helps identify fine structure in the contributions of the "intrinsic" and "extrinsic" parts of the transistor to the overall

Manuscript received May 11, 2007; revised July 6, 2007. This work was supported by the Natural Sciences and Engineering Research Council of Canada. The work of D. L. John was supported by Research Councils UK. The review of this paper was arranged by Associate Editor M. Anantram.

D. L. Pulfrey is with the Electrical and Computer Engineering Department, University of British Columbia, Vancouver, BC V6T 1Z4, Canada.

L. C. Castro was with the University of British Columbia, Vancouver, BC V6T 1Z4, Canada. He is now with Qimonda, Munich, Germany.

D. L. John is with the School of Electronic Engineering, University of Wales, Bangor LL57 1UT, U.K.

M. Vaidyanathan is with the Electrical and Computer Engineering Department, University of Alberta, Edmonton, AB T6G 2V4, Canada.

Color versions of one or more of the figures in this paper are available online at http://ieeexplore.ieee.org.

Digital Object Identifier 10.1109/TNANO.2007.907796

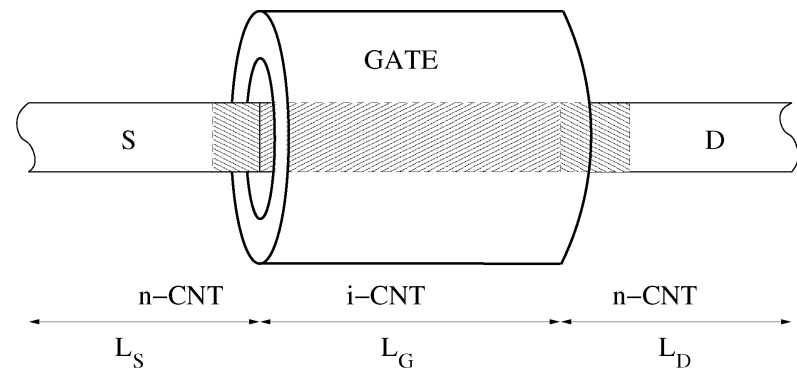

Fig. 1. Schematic of $n-i-n$ CNFET with wrap-around gate and semi-infinite source- and drain-contacts. The shading indicates regions within the $n$-doped source and drain portions of the nanotube, and within the initially intrinsic channel, where there are changes in space charge in response to a change in gate potential.

signal delay time, and hence to $f_{T}$. It also allows, in this instance, elucidation of the relationship between the propagation velocity of electrons carrying the conduction current, and the signal velocity. The former is a physical property determined by the band structure of the material, whereas the latter relates directly to the charge-control definition of $f_{T}$. Clarification of this issue is important in view of the apparent breeching by recent simulation results [7], [8] of a suggested minimum limit for the signal delay time [9].

The doped-contact $n-i-n$ CNFET is used for this study in view of the very high values of $f_{T}(\approx 50 \mathrm{THz})$ that have been predicted for this device [7], [8]. However, the proposed signaldelay analysis is applicable to ballistic FETs other than just the CNFETs considered here.

\section{REgiOnAL SignAL-DELAY ANALYSIS}

\section{A. Derivation of Delay Time}

Applying charge-control theory [10] to FETs, the overall, source-drain signal delay time $\tau_{\mathrm{SD}}$ relates to $f_{T}$, and to the change in input charge that is required to bring about a change in output current

$$
\tau_{\mathrm{SD}}=\frac{1}{2 \pi f_{T}}=\frac{\partial Q}{\partial I}
$$

where, for a FET, $\partial Q=\partial Q_{\text {in }}=\partial Q_{G}$ is the change in charge on the gate, and $\partial I=\partial I_{\text {out }}=\partial I_{D}$ is the change in drain current. Here, we consider the changes in charge and current due to a change in gate-source voltage $\partial V_{\mathrm{GS}}$. The CNFET is schematically depicted in Fig. 1: the continuous nanotube is doped in the source and drain portions, and the channel is intrinsic. Far away from the interfaces between the doped and intrinsic regions, the source and drain leads are charge neutral, 
and, therefore, we examine the charge change in the entire nanotube $\partial Q_{C N}=-\partial Q_{G}$, as this allows access to the regional delay times. Also, there is no hole current in this arrangement, so $\partial I_{D}=-\partial I_{n}$, where $I_{n}$ is the electron current. The derivatives are appropriately written as

$$
\begin{aligned}
\partial Q & =\int_{z} \int_{E} \partial Q(z, E) d E d z \\
\partial I & =\int_{E} \partial I(E) d E .
\end{aligned}
$$

By examining different regions $r$ of the device, regional signal delays $\tau_{r}$ can be defined

$$
\tau_{\mathrm{SD}}=\sum_{r}^{L_{\mathrm{CNQ}}} \tau_{r}=\frac{1}{\partial I} \sum_{r}^{L_{\mathrm{CNQ}}} \int_{r} \partial Q(z) d z
$$

where $L_{\mathrm{CNQ}}$ is the length of the nanotube over which there is a change in charge, and $\partial Q(z)$ is the local charge density integrated over energy, i.e.,

$$
\partial Q(z)=\int_{E} \partial Q(z, E) d E .
$$

In regions where there is no charge change, the charge-control approach gives $\tau_{r}=0$. In reality, the appropriate delay time in these regions is the dielectric relaxation time. Here, such regions are the quasi-neutral portions of the source and drain contacts.

\section{B. Definition of Signal Velocity}

Dividing the local charge change by the spatially independent current change allows a local signal velocity to be defined

$$
\frac{1}{v_{\mathrm{sig}}(z)}=\frac{\partial Q(z)}{\partial I}
$$

Clearly, the local signal velocity and the regional signal delay are related

$$
\tau_{r}=\int_{r} \frac{1}{v_{\mathrm{sig}}(z)} d z
$$

\section{Relation Between $v_{\mathrm{sig}}$ and Propagation Velocity}

Fig. 2 attempts to portray the energetic environment experienced by an electron propagating between source and drain. In our analysis the effective-mass form of the Schrödinger equation is employed, and the potential profile is represented by a piece-wise-rectangular approximation, as shown schematically in the figure. Under these circumstances, the probability density current gives the propagation velocity as $\hbar k / m$. This is the band-structure-determined velocity $v_{b}=\partial E / \hbar \partial k$; it enters into the analysis via the change in conduction current $\partial I$.

This current is spatially invariant, and so is $\partial I(E)$ in the ballistic case. So, for source-originating electrons, as shown in Fig. 2, we elect to describe their contribution to the current deep in the drain, at some point $z=D$, where $v_{b}$ will attain its highest

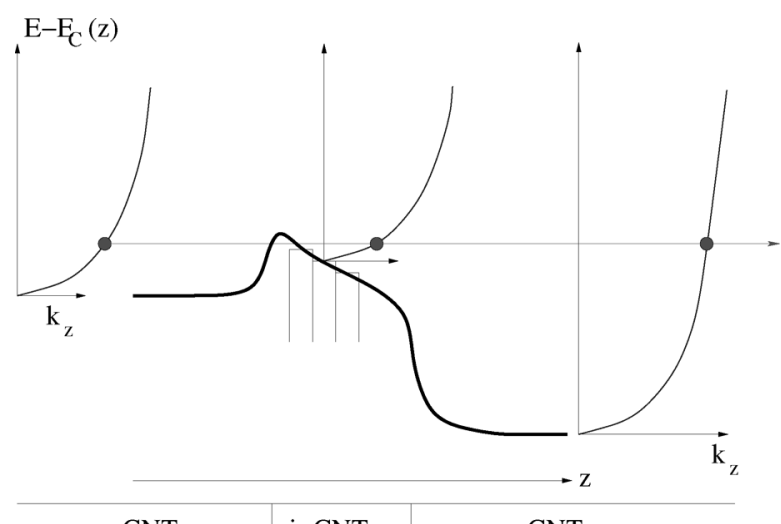

Fig. 2. Montage of conduction-band (first subband) edge and kinetic energy $v s$ longitudinal wavevector for a $n-i-n$ CNFET with semi-infinite source- and draincontacts. Part of the piece-wise rectangular representation of the conductionband profile is also shown. An electron in transit is depicted. Note that the kinetic energy, and hence the propagation velocity, is highest deep in the drain.

value. Similarly, the current due to drain-originating electrons is described deep in the source, at some point $z=S$. Thus, the total change in current is

$$
\partial I=\int_{E} \partial\left\{v_{b}(D, E) Q_{S}(D, E)-v_{b}(S, E) Q_{D}(S, E)\right\} d E
$$

where, for example, the source-originating electronic charge is

$$
Q_{S}(z, E)=-q \mathcal{G}_{S}(z, E) f_{S}(E)
$$

where $\mathcal{G}_{S}$ is the local density of states (LDOS) arising from coupling to the source contact, for which the Fermi level is $f_{S}(E)$. The actual LDOS is

$$
\mathcal{G}_{S}(z, E)=g_{S}(E) \psi_{S}(z, E) \psi_{S}^{*}(z, E)
$$

where $g_{S}(E)$ is the nonlocal density of states in the essentially semi-infinite, neutral region of the source contact, and $\psi_{S}$ is the wavefunction of electrons issuing from this contact. Similar expressions are used for electrons originating in the drain contact. The wavefunctions in this open system are normalized by equating the probability density current and the Landauer current [12].

It is reasonable to assume, particularly at high drain-source bias $V_{\mathrm{DS}}$, that there is no contribution to $\partial I$ from drain-injected electrons. Thus, (7) can be simplified to

$$
\partial I=\bar{v}_{\text {band }}(D) \int_{E} \partial Q_{S}(D, E) d E
$$

where $\bar{v}_{\text {band }}(D)$ is the average propagation velocity deep in the drain of the electrons that carry $\partial I$, i.e.,

$$
\bar{v}_{\text {band }}(D)=\frac{\int_{E} \partial\left\{v_{b}(D, E) Q_{S}(D, E)\right\} d E}{\int_{E} \partial\left\{Q_{S}(D, E)\right\} d E} .
$$

For some carbon nanotubes the chirality is such that the $E-k$ dispersion relation is linear over some region of energy and, further, that the constant $v_{b}$ in this region is maximal at the putative 
(a)

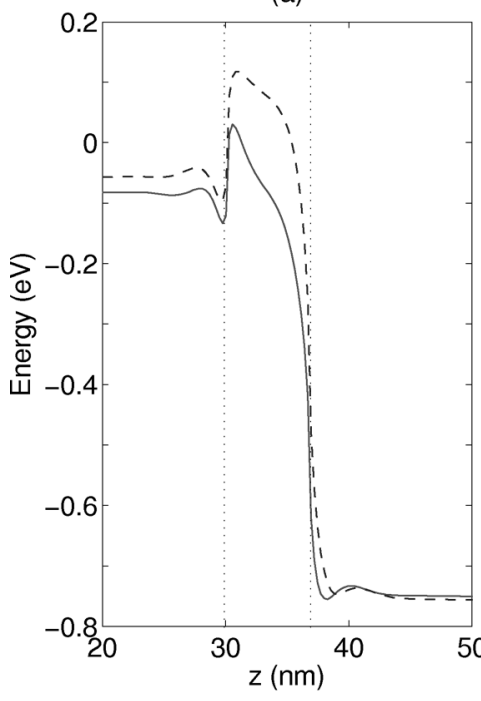

(b)

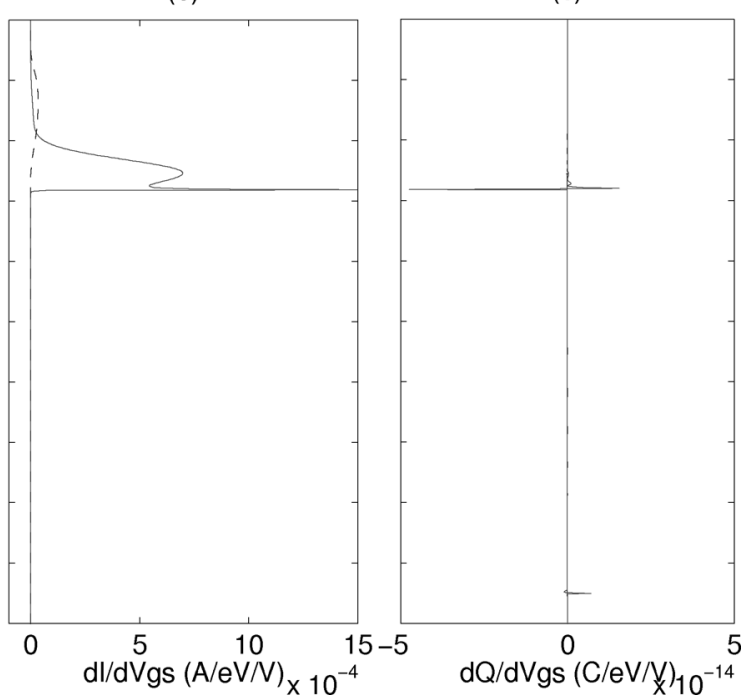

Fig. 3. Composite figure for $V_{\mathrm{DS}}=0.7 \mathrm{~V}$ and $V_{\mathrm{GS}}=0.4 \mathrm{~V}$ (dashed line) and $V_{\mathrm{GS}}=0.7 \mathrm{~V}$ (solid line). The energy reference is the source Fermi level. (a) Conduction band (lowest subband) diagram. The channel extends from $z=30-37 \mathrm{~nm}$, as delineated by the dotted lines. (b) Change in spectral current for $\partial V_{\mathrm{GS}}=+5 \mathrm{mV}$. (c) Change in spectral charge for $\partial V_{\mathrm{GS}}=+5 \mathrm{mV}$. Note: on this scale the change in spectral charge for $V_{\mathrm{GS}}=0.4 \mathrm{~V}$ is not visible, nor can much quantitative information be gleaned for the $V_{\mathrm{GS}}=0.7 \mathrm{~V}$ case. The figure is included merely to illustrate the very small energy ranges over which the charge changes.

Fermi velocity. Here, because we are using an effective-mass formalism, the high-velocity electrons in the drain will be spread over a small energy range. Nevertheless, we take $\bar{v}_{\text {band }}(D)$ as being representative of the highest propagation velocity attainable in our case, i.e.,

$$
v_{b, \text { high }} \equiv \bar{v}_{\text {band }}(D) .
$$

Substituting (12) into (10), and in turn into (5), a relation between signal velocity and the highest propagation velocity emerges

$$
v_{\text {sig }}(z)=v_{b, \operatorname{high}} \frac{\partial Q_{S}(D)}{\partial Q(z)}
$$

where $Q(z)=Q_{S}(z)+Q_{D}(z)$ is the total local charge density, i.e., it includes the contributions from both the source- and drain-injected electrons.

Clearly, $v_{\text {sig }}(z)$ is not limited by $v_{b, \text { high }}$ because $v_{\text {sig }}(z) \rightarrow \infty$ in the quasi-neutral regions. However, the question that needs to be answered regarding very high performance FETs is: can $v_{\text {sig }}(z)>v_{b, \text { high }}$ be sustained over the space-charge regions? In particular, can $v_{\mathrm{sig}}(z)>v_{b}$,high arise over much of the channel? If the answer is affirmative, then the limit to the delay in the channel, $\tau_{G}$, of $L_{G} / v_{b \text {,high }}$, as suggested in [9], could be breeched, thereby providing a possible explanation for the extremely short delay time implicit in the results of [7]. Equation (13) does not readily provide a direct answer to these questions because of the difficulty in guessing how $Q(z)$ changes. This is particularly true in the short channel of a high-performance device, where quantum-mechanical reflections and resonances will lead to changes in quasi-bound state densities that will be different from $\partial Q_{S}(D)$. Evidently, numerical simulations are needed to elucidate the situation.

\section{RESULTS AND DISCUSSION}

Results are presented from simulations of a coaxial, $n-i-n$ CNFET, with $L_{S}=L_{D}=30 \mathrm{~nm}, L_{G}=7 \mathrm{~nm}$, and an oxide of thickness $2 \mathrm{~nm}$ and relative permittivity 3.9. These properties are similar to those used in the simulation study that produced the record- $f_{T}$ prediction [7], [8], although that transistor had double planar gates, rather than a coaxial gate. The lengths of our doped regions were extended to $30 \mathrm{~nm}$ to ensure that all the charge change due to a change in gate bias of $5 \mathrm{mV}$ was encompassed. Thus, we are using the quasi-static approach for determining $f_{T}$; this is justified since this figure-of-merit is defined by extrapolation from low-frequency data and hence it is, in fact, a quasi-static parameter [10]. The doping density in the source and drain regions was $5 \times 10^{8} \mathrm{~m}^{-1}$, which corresponds to a dopant molar fraction of $\approx 5 \times 10^{-3}$. This positive charge is represented by a surface charge, distributed uniformly around the source and drain portions of the nanotube [11]. Self-consistent simulations, including both electrons and holes, were performed using an effective-mass Schrödinger-Poisson solver [12], with an effective mass of $0.122 m_{0}$, as befits an $(11,0)$ tube. As is now customary in systems comprising heavily-doped contacts, and for which ballistic transport applies, floating boundary conditions were employed [13], and only the lowest subband was considered [14]. $V_{\mathrm{DS}}$ was $0.7 \mathrm{~V}$, and the range of $V_{\mathrm{GS}}$ was $0.4-0.7 \mathrm{~V}$.

A sample energy band diagram (lowest subband for electrons) is shown in Fig. 3(a). The following features are noteworthy: "dips" in potential in the doped portions of the tube proximal to the gated region; a large potential barrier at the junction with the doped source; a strong field in the channel. The first feature is related to the strong fringing fields emanating from the gate, due to its wrap-around nature and the small oxide thickness. Additionally, potential variations in the doped regions near to the gate can be expected due to ripples in the charge density itself: this is an interference phenomenon, arising from the reflection of 


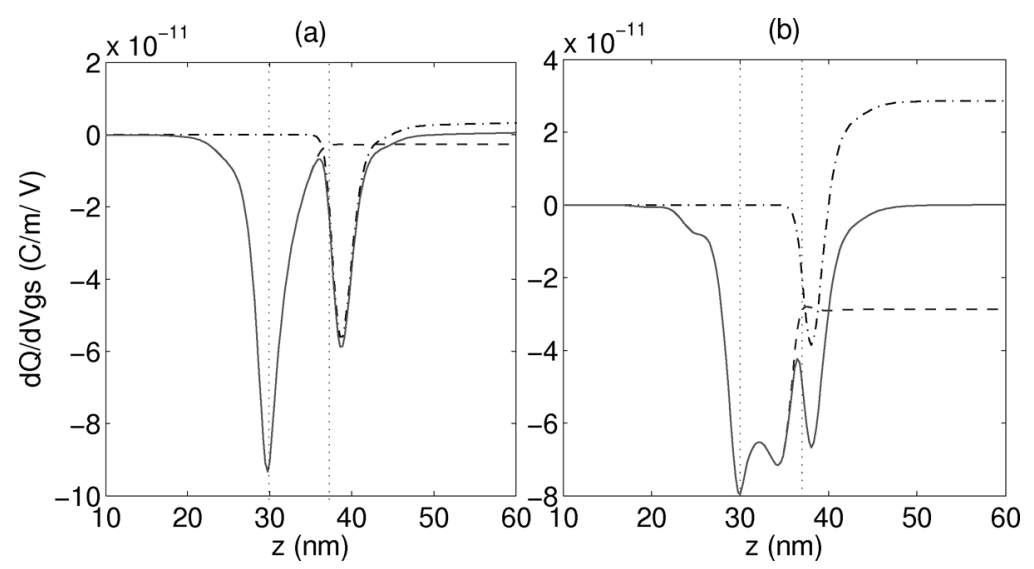

Fig. 4. Change in the source- and drain-originating components of the spatial charge for $\partial V_{\mathrm{GS}}=+5 \mathrm{mV}$ at $V_{\mathrm{DS}}=0.7 \mathrm{~V}$. Total charge change (solid line), $\partial Q_{S}$ (dashed line), $\partial Q_{D}$ (stippled line). The channel extends from $z=30-37 \mathrm{~nm}$, as marked by the dotted lines. (a) $V_{\mathrm{GS}}=0.4 \mathrm{~V}$. (b) $V_{\mathrm{GS}}=0.7 \mathrm{~V}$.

electrons incident from the contact regions. Regarding the high potential barrier at the source, this is indicative of an insensitivity to changes in $V_{\mathrm{GS}}$, brought about by the local capacitance due to the charge at the source end of the channel being greater than the oxide capacitance. The peaked nature of the potential profile at the source end of the channel is exacerbated by the proximity of the drain. In CNFETs with longer channels, the potential profile is smoother [11].

The change in current due to a small-signal change of $+5 \mathrm{mV}$ in the gate potential is shown in Fig. 3(b): it can be seen from the spectral range of this difference current that tunneling through the barrier at the source/channel interface is involved, particularly for the case of $V_{\mathrm{GS}}=0.7 \mathrm{~V}$. The corresponding change in spectral charge is shown in Fig. 3(c). Note that the majority of this charge change occurs at energies possessed by electrons issuing from the source. The small charge change at lower energies is due to changes in the charge injected from the drain $\partial Q_{D}$. At low $V_{\mathrm{GS}}$ almost the entire charge change in the drain "depletion region" is due to $\partial Q_{D}$, as is clear from the plot of spatial charge change in Fig. 4(a). At higher bias, source-originating charge is also present in this region [see Fig. 4(b)]. At both biases there is some penetration into the bandgap of evanescent waves from the drain. Deep in the drain, to maintain charge neutrality, $\partial Q_{D}$ is positive, representing the loss of electrons necessary to compensate for the arrival of electrons from the source.

The spatial change of the source-originating electron charge is also shown in Fig. 4. This charge, $\partial Q_{S}$, increases (negatively) to reduce the width of the depletion region in the source, and to supply the charge needed to maintain the current in the presence of the retarding, tunneling barrier. The charge density then rarefies as acceleration occurs in the field [see Fig. 3(a)]. In the higher bias case of Fig. 4(b), a secondary charge peak is seen at about $z=34 \mathrm{~nm}$. Deep in the drain the source-originating difference charge $\partial Q_{S}(D)$ settles to a constant value, commensurate with flow in a uniform, field-free region.

The actual signal delays $\tau_{S}, \tau_{G}$, and $\tau_{D}$ for the geometrically defined regions of the source, channel (gated region), and the drain, respectively, are shown in Fig. 5. Each of these components decreases with increasing $V_{\mathrm{GS}}$. At the highest bias examined, the source- and drain-delays account for about $50 \%$ of the

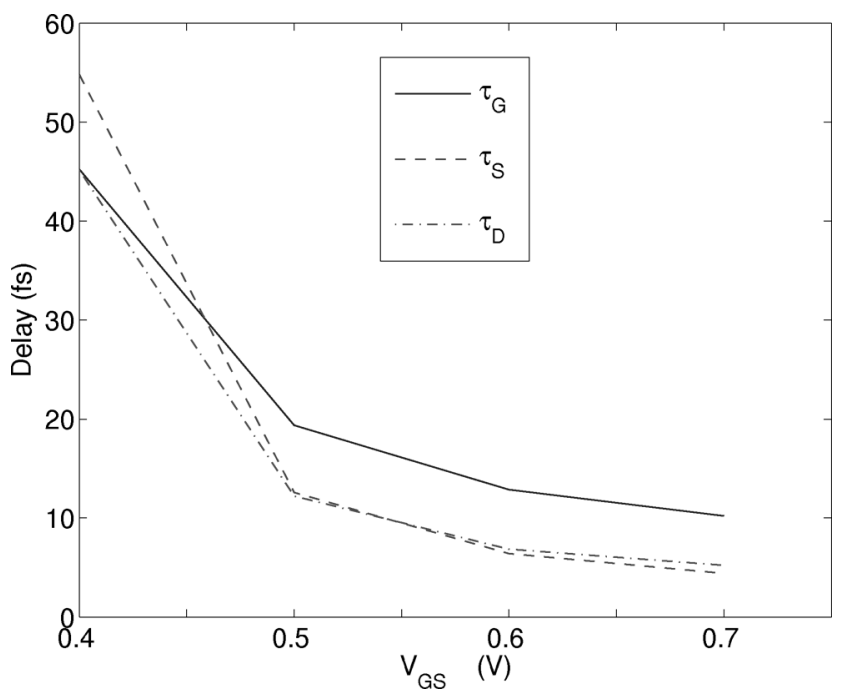

Fig. 5. Gate-bias dependence of the signal delays for the geometric regions of the source, channel (the gated region), and the drain. $V_{\mathrm{DS}}=0.7 \mathrm{~V}$.

overall delay of $21 \mathrm{fs}$. A finer breakdown of the regional signal delays at high bias is shown in Fig. 6. The largest contribution comes from the region in the channel where a potential barrier is presented to the charge flow $\partial I$. This highlights the fact that tunneling in these devices causes a serious bottleneck to charge transport. In the second half of the channel the delay is less, as would be expected from the acceleration of the electrons by the field (see Fig. 6, top panel). Fig. 6 also shows regional delays due to changes in the charge from the drain $\partial Q_{D}$. The results quantify the observations noted with respect to Fig. 4: the delay due to the inevitable modulation of the depletion region width at the channel/drain junction is much larger than that due to the filling of evanescent states in the channel. $\tau_{D}$ may be underestimated here because of our neglect of electron-phonon interactions: kinetic energies as high as $\approx 1 \mathrm{eV}$ are possible in the drain, at which optical- and zone-boundary-scattering may be important [15]. Underestimation of $\tau_{D}$ may also be occurring due to overestimation of $v_{b}$ by our effective-mass treatment. Comparison with results from Tight-Binding calculations for an $(11,0)$ tube indicate an overestimate of $v_{b}$ by about a factor of 2 at a kinetic energy of $\approx 1 \mathrm{eV}$. This will have some impact on $v_{\mathrm{sig}}(D)$ via 


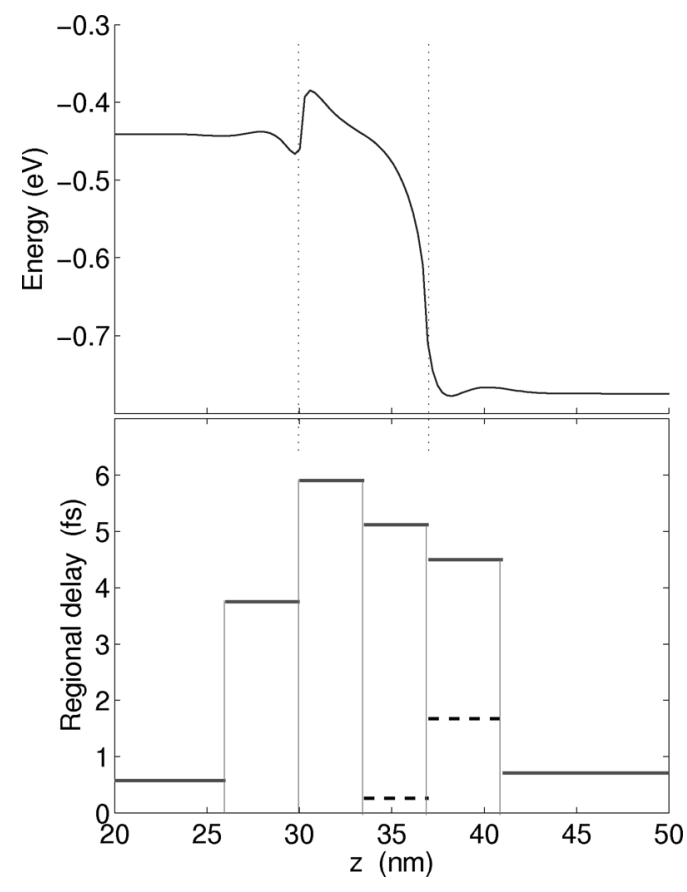

Fig. 6. Regional signal delays (bottom), registered to the conduction band profile (top). The contributions to the total delays (solid lines) by the delays due to $\partial Q_{D}$ are depicted by the dashed lines. The channel extends from $z=30-$ $37 \mathrm{~nm}$, as marked by the dotted lines. $V_{\mathrm{DS}}=V_{\mathrm{GS}}=0.7 \mathrm{~V}$.

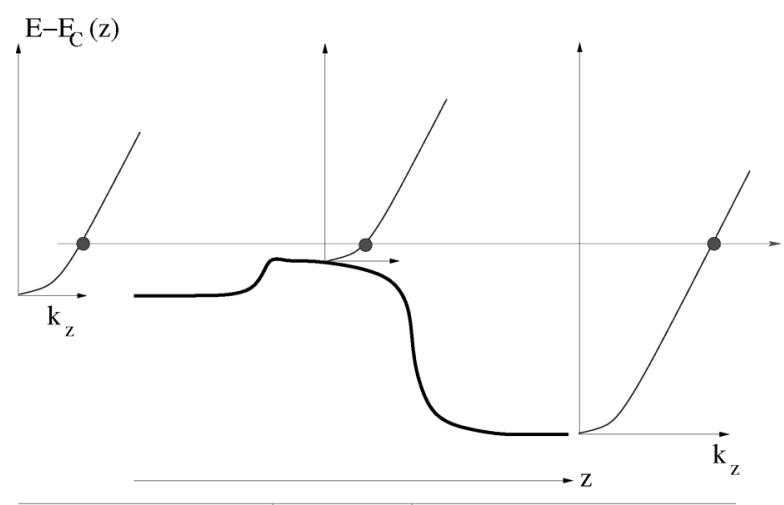

\begin{tabular}{l|l|l} 
n-CNT & i-CNT & n-CNT
\end{tabular}

Fig. 7. Similar to Fig. 2, except that: the dispersion relation now shows an extensive linear region; the signal current is carried by electrons in states within this linear region of $E-k$; and there is no tunneling.

(13). Elsewhere in the device, where kinetic energies are much lower, there should be little error, as the calculations of $v_{b}$ by the two methods are in better agreement.

We now wish to examine the recently proposed limit to the signal delay time in the gate [9]: $\tau_{G, \min }=L_{G} / v_{b, \text { high }}$. There are two necessary conditions that must be met before this limit can be seriously considered: the signal velocity must equal the highest band-limited velocity; and the latter must be constant across the channel. Fig. 7 depicts a situation in which $v_{b \text {,high }}$ might be sustained over the entire channel. This situation differs from that shown in Fig. 2 in three ways: the $E-k$ dispersion relation has an extensive linear region, signifying a constant $v_{b \text {,high }}$; the signal current is carried by electrons in states for which this maximum velocity applies; and there is no tunneling. The realism of this situation is debatable, but let us move on to

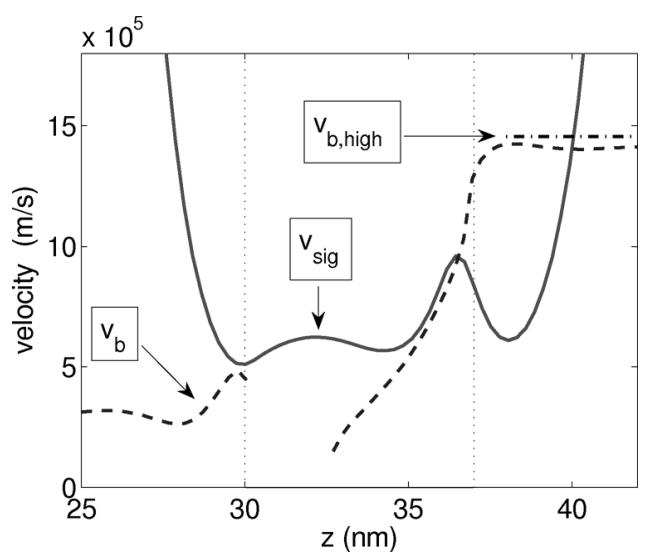

Fig. 8. Velocities at $V_{\mathrm{DS}}=V_{\mathrm{GS}}=0.7 \mathrm{~V}$. Solid line is $v_{\mathrm{sig}}$, and dashed line is $v_{b}$ evaluated at the energy of the broad peak in $\partial I(E)$ in Fig. 3(a). The stippled line is $\bar{v}_{\text {band }}(D)$ from (11). The channel extends from $z=30-37 \mathrm{~nm}$, as marked by the dotted lines.

consider the other necessary condition of $v_{\text {sig }}(G)$, the signal velocity in the channel, being equal to $v_{b \text {,high }}$. The relevant relationship is given in (13), where the source-originating charge of the numerator represents the unidirectional charge flow in the drain; the source-originating charge in the denominator, when applied to the channel, has both drain-directed and reflected components. Additionally, the denominator includes a contribution from the drain-injected electrons. It is clear from this equation that one way in which the signal velocity in the channel could equal $v_{b \text {,high }}$ would be if $\partial Q_{D}(G) \ll \partial Q_{S}(G)$, and if $\partial Q_{S}(G)$, the change in source charge in the channel, were to represent a unidirectional flow equal to that in the drain. These restrictions are consistent with Fig. 7, with the additional qualifications that there must be no reflections from the potential steps at the ends of the channel, and that there be insignificant charge injected from the drain into the channel. All the above conditions were met by the semiclassical analysis of [9].

In the present, quantum-mechanical study, we have the opportunity to examine the effect of the removal of all of the above conditions and restrictions, at least for the particular device considered. Firstly, there is tunneling, particularly at higher $V_{\mathrm{GS}}$, so the propagation velocity will be less than $v_{b \text {,high }}$ in parts of the channel. There is some injection of charge from the drain, but it is minor compared to that injected from the source. Electrons constituting the latter charge will be reflected from the ends of the channel, leading to resonances and spatial variation in the LDOS. It is conceivable that the change in LDOS, due to the change in gate bias, could result in local regions within the channel having very low values of $\partial Q_{S}(z)<\partial Q_{S}(D)$. However, such regions would inevitably be juxtaposed with regions of high values of LDOS (nodes and antinodes), so it is difficult to see how $\partial Q_{S}(z)$ over the entire channel could be very low. Certainly, in the present work, there is no evidence of this occurring, as can be seen from Fig. 8, which shows $v_{\text {sig }}(z)$ as being much less than $v_{b \text {,high }}$ throughout the channel.

Also shown in Fig. 8 is the propagation velocity $v_{b}$ for electrons at $E \approx-0.05 \mathrm{eV}$, i.e., at the energy of the broad peak of the differential current shown in Fig. 3(a). In the tunneling region of the first part of the channel, the propagation velocity is not well-defined, but it can be seen that it climbs towards 
the signal velocity in the second half of the channel. There is a high field there, somewhat reminiscent of the situation in the base-collector space-charge region of a BJT. In the latter region it is well-known that $v_{\text {sig }}$ can exceed $v_{b}[16]$, although the difference diminishes below the usual factor of 2 when the movement is from a low-field region to a high-field region, as is the case in our device. However, the difference between the two velocities in the second part of the channel is not really significant here, and, in any case, direct analogies with the BJT case are dubious, on account of the complex electrostatics due to the two-dimensional arrangement of the electrodes in FETs [17].

In summary, there is no evidence from this study that the semiclassical limit to the delay time in the channel can be breeched. This result is contrary to that which can be inferred from the simulations reported in [7] and [8]. The device in that work was not too dissimilar from that studied here: it had a $7 \mathrm{~nm}$ channel and $10 \mathrm{~nm}$ extensions for the source and drain contacts. The predicted $f_{T}$ at biases comparable to those used here was $\approx 47 \mathrm{THz}$. This value, which is about 6 times larger than our value, and two orders of magnitude higher than the target value for $\mathrm{Si}$ MOSFETs intended for high-performance $\mathrm{RF}$ applications in 2014 [18], implies an overall signal delay time of $3.4 \mathrm{fs}$. To get a lower bound on the average signal velocity in the channel for this device, let us divide the gate length by this delay time. The result is $v_{\text {sig }}(G) \approx 2 \times 10^{6} \mathrm{~m} / \mathrm{s}$. This is even higher than the values of $v_{b}$,high computed here (see Fig. 8), and our velocities are already high because of the effective-mass approximation. This suggests not only an absence of tunneling, but also a presence of phenomena that boost $v_{\text {sig }}$, and which are not evident in the present study. Further work is required to explain this extraordinarily short delay time, and the associated, implied, very high signal velocity in the channel.

\section{CONCLUSION}

From this simulation study of high-frequency FETs it can be concluded that:

i) regional signal-delay analysis, which has long been used to guide the design of BJTs, can be usefully employed to study high-frequency FETs;

ii) in the carbon nanotube FET studied here, the presence of a tunneling barrier at the entry to the channel region causes a large signal delay;

iii) the signal delay times in the doped portions of the nanotube, proximal to the channel, also contribute significantly to the overall delay (about $50 \%$ at the highest bias used in this example);

iv) the relevant velocity for $f_{T}$ calculations is not the propagation velocity: it is the signal velocity, which we have defined;

v) the signal velocity in the channel does not, at least in the device studied here, exceed the highest propagation velocity, which is attained in the drain;

vi) the quantum-mechanical effect of resonances within the channel does not cause a breeching of the semiclassical limit for the minimum signal delay in the channel.

\section{Note Added in Proof:}

During the processing of this paper, the authors of [7] and [8] communicated to D. L. Pulfrey that their value of $f_{T}=47 \mathrm{THz}$, as reported in their papers, is to be replaced by $f_{T}=9 \mathrm{THz}$. This prediction does not breach the limit proposed in [9], and is consistent with our own results.

\section{ACKNOWLEDGMENT}

The authors would like to thank Drs. N. Moll and J. Guo for helpful comments on image charges in multidimensional systems and on band-edge "dips," respectively.

\section{REFERENCES}

[1] A. Le Louarn et al., "Intrinsic current gain cutoff frequency of 30 GHz with carbon nanotube transistors," Appl. Phys. Lett., vol. 90, pp. 233108-1-233108-3, 2007.

[2] B. C. Paul, S. Fujita, M. Okajima, and T. Lee, "Impact of geometry-dependent parasitic capacitances on the performance of CNFET circuits," IEEE Electron Device Lett., vol. 27, no. 5, pp. 380-382, May 2006.

[3] D. L. John and D. L. Pulfrey, "Switching-speed calculations for schottky-barrier carbon nanotube field-effect transistors," J. Vac. Sci. Technol., vol. 24, pp. 708-712, 2006.

[4] J. Guo, A. Javey, H. Dai, and M. Lundstrom, "Performance analysis and design optimization of near-ballistic carbon nanotube field-effect transistors," IEDM Tech. Dig., pp. 703-706, 2004.

[5] H. F. Cooke, "Microwave transistors: Theory and design," Proc. IEEE, vol. 59, no. 8, pp. 1163-1181, Aug. 1971.

[6] W. Hafez and M. Feng, "Experimental demonstration of pseudomorphic bipolar transistors with cutoff frequencies above $600 \mathrm{GHz}$," $\mathrm{Appl}$. Phys. Lett., vol. 86, no. 15, pp. 152101-1-152101-3, Apr. 2005.

[7] G. Fiori, G. Iannaccone, and G. Klimeck, "Performance of carbon nanotube field-effect transistors with doped source and drain extensions and arbitrary geometry," in IEDM Tech. Dig., 2005, pp. 522-525.

[8] G. Fiori, G. Iannaccone, and G. Klimeck, "A three-dimensional simulation study of the performance of carbon nanotube field-effect transistors with doped reservoirs and realistic geometry," IEEE Trans. Electron Devices, vol. 53, pp. 1782-1788, 2006.

[9] S. Hasan, S. Salahuddin, M. Vaidyanathan, and M. A. Alam, "High-frequency performance projections for ballistic carbon-nanotube transistors," IEEE Trans. Nanotechnol., vol. 5, pp. 14-22, 2006.

[10] H. K. Gummel, "On the definition of the cutoff frequency $f_{t}$," Proc. IEEE, vol. 57, no. 12, p. 2159, Dec. 1969.

[11] G. Fiori, G. Iannaccone, M. Lundstrom, and G. Klimeck, "Three-dimensional atomistic simulation of carbon nanotube fets with realistic geometry," in Proc. ESSDERC, 2005, pp. 537-540.

[12] D. L. John, L. C. Castro, P. J. S. Pereira, and D. L. Pulfrey, "A schrödinger-poisson solver for modeling carbon nanotube fets," in Tech. Proc. 2004 Nanotechnology Conf. Trade Show, , vol. 3, pp. 281-286.

[13] R. Venugopal, Z. Ren, and M. S. Lundstrom, "Simulating quantum transport in nanoscale mosfets: Ballistic hole transport, subband engineering and boundary conditions," IEEE Trans. Nanotechnol., vol. 2, pp. 135-143, 2003.

[14] G. Fiori and G. Iannaccone, "Threshold voltage dispersion and impurity scattering limited mobility in carbon nanotube field-effect transistors with randomly doped reservoirs," in Proc. ESSDERC, 2006, pp. 202-205.

[15] Y. Yoon, Y. Ouyang, and J. Guo, "Effect of phonon scattering on intrinsic delay and cutoff frequency of carbon nanotube fets," IEEE Trans. Electron Devices, vol. 53, no. 10, pp. 2467-2470, Oct. 2006.

[16] S. E. Laux and W. Lee, "Collector signal delay in the presence of velocity overshoot," IEEE Electron Device Lett., vol. 11, no. 4, pp. 174-176, Apr. 1990.

[17] P. Roblin and H. Rohdin, High-Speed Heterostructure Devices. Cambridge, U.K.: Cambridge Univ. Press, 2002, sec. 15.4.

[18] International Technology Roadmap for Semiconductors 2006 [Online]. Available: http://www.itrs.net/links/ 2006Update/FinalToPost/05_Wireless2006Update.pdf 


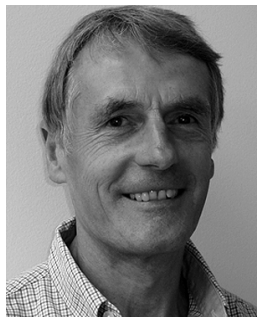

David Pulfrey (M'73-S'90-F'00) received the B.Sc. and $\mathrm{Ph} . \mathrm{D}$. degrees in electrical engineering from the University of Manchester, U.K., in 1965 and 1968, respectively.

Since 1968 he has been on the faculty in the Electrical Engineering Department at the University of British Columbia, Vancouver, Canada. He is the author of many papers and two books: Photovoltaic Power Generation (Van Nostrand Reinhold, 1979), and, with G. Tarr, Introduction to Microelectronic Devices (Prentice-Hall, 1989). His research area is semiconductor device modeling, presently with emphasis on numerical analyses of, and compact models for, carbon nanotube transistors. Previously, he developed models for MIS tunnel junctions, HBTs, photodetectors, and solar cells.

Dr. Pulfrey was the inaugural winner of UBC's Teaching Prize for Engineering in 1990. He was elected a Fellow of the IEEE in 2000 for contributions to the modeling of heterojunction bipolar semiconductor devices, and a Fellow of the Canadian Academy of Engineering in 2003 for outstanding contributions to teaching and research in microelectronics.

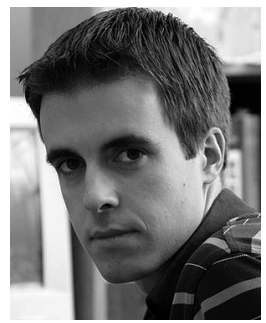

Leonardo Castro received the B.A.Sc. and Ph.D. degrees in electrical engineering from the University of British Columbia, Canada, in 2001 and 2006, respectively.

He has been working since 2006 on DRAM development at Qimonda, Munich, Germany. At university his research activities were in carbon nanotube transistor modeling, with particular emphasis on predictions of high-frequency performance.

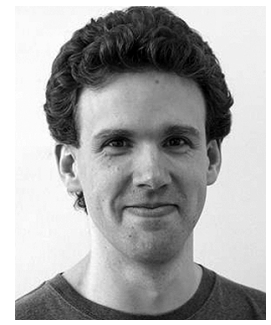

David John (S'01-M'06) received the B.A.Sc. degree (Hons.) in engineering physics and the Ph.D. degree in electrical engineering from the University of British Columbia, Canada, in 2002 and 2006, respectively.

He is presently an RCUK Academic Fellow at the University of Wales, Bangor.

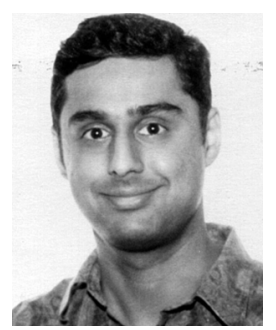

Mani Vaidyanathan received the B.A.Sc. degree in computer engineering (including co-op work terms with firms such as Nortel Networks, IBM, and Genesis Microchip) and the M.A.Sc. degree in electrical engineering from the University of Waterloo (UW), Canada, in 1990 and 1993, respectively, and the Ph.D. degree from the University of British Columbia (UBC), Canada, in 1999.

From 1993 through 1994 he was an Adjunct Lecturer and Research Assistant at UW, teaching courses and performing research in the area of semiconductor devices. He began his dissertation work at UBC in 1994. In 1999, he joined the Department of Electrical and Computer Engineering at the University of California at San Diego (UCSD), La Jolla, CA, as a Postdoctoral Fellow, and was promoted to Assistant Research Scientist in Fall 2001. During 2002-2003, he was on leave from UCSD and held the position of Visiting Assistant Professor at Purdue University, West Lafayette, IN, and in Fall 2004, he joined the faculty of the University of Alberta, Edmonton, Canada, where he is currently an Assistant Professor. His research interests are in the theory and modeling of semiconductor devices, where he has worked on topics ranging from studying carrier transport in small-dimension devices to modeling high-frequency distortion for wireless applications.

Dr. Vaidyanathan received postgraduate scholarships from the Natural Sciences and Engineering Research Council (NSERC) of Canada for the M.A.Sc. and $\mathrm{Ph} . \mathrm{D}$. degrees, as well as an NSERC postdoctoral fellowship. He was a Killam Scholar and Governor-General's Gold Medal nominee at UBC, and received the Sandford Fleming Award for excellence in teaching assistantship at UW. 